九州大学学術情報リポジトリ

Kyushu University Institutional Repository

\title{
SELECTING BETTER GENERIC DRUGS
}

\section{Hayakawa, Masanobu}

Graduate School of Medicine, Kurume University

Yanagawa, Takashi

Biostatistics Center Kurume University

Jiang, Ying

Institute of Industrial Ecological Sciences, University of Occupational and Environmental Health

Sakurai, Rieko

Graduate School of Medicine, Kurume University

https://doi.org/10.5109/1798147

出版情報: Bulletin of informatics and cybernetics. 46, pp.53-70，2014-12. Research Association of Statistical Sciences

バージョン :

権利関係 : 


\title{
SELECTING BETTER GENERIC DRUGS
}

\author{
by \\ Masanobu HaYakawa, Takashi Yanagawa, Ying Jiang \\ and \\ Rieko SAKURAI
}

Reprinted from the Bulletin of Informatics and Cybernetics

Research Association of Statistical Sciences, Vol. 46

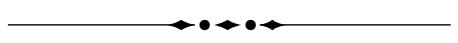

FUKUOKA, JAPAN

2014 


\title{
SELECTING BETTER GENERIC DRUGS
}

\author{
By \\ Masanobu HaYaKawa* Takashi Yanagawa $\stackrel{\text { Ying JIANG }}{\ddagger}$ \\ and \\ Rieko SAKURAI ${ }^{\S}$
}

\begin{abstract}
Often, more than twenty generic (GE) drugs are developed for one brand name (B-N) drug. Each GE drug has passed the trial of bio-equivalence to the B-N drug, and its characteristics (which are generally expressed by AUC, Cmax, and Tmax, or one of its subsets) are close to the values for the B-N drug in each trial. However, trial-to-trial variation in the AUC, Cmax, and Tmax values is substantial. A model is developed in this paper to extract the distance between the characteristics of the GE and B-N drugs by adjusting for trial-to-trial variation. A method is proposed in this paper to use this distance for selecting better GE drugs from the list of candidates.
\end{abstract}

Key Words and Phrases: Brand-name drug, Bio-equivalence, Cross-over design, Random effect model.

\section{Introduction}

Generic (GE) drugs are drugs that are bio-equivalent to a brand name (B-N) drug and have been developed after the termination of the patent of the B-N drug. Often there are twenty or more GE drugs developed for each B-N drug. For example, for Amlodipine besylate, the B-N drug to treat hypertension and angina, 27 GE drugs are on sale in the market in the US and 40 are on sale in Japan as of September, 2012. The approval and license for production and sale of a GE drug are given to companies by a country's drug administration after confirming the bio-equivalence of the drug to the $\mathrm{B}-\mathrm{N}$ drug. AUC and Cmax are used for testing bio-equivalence. A brief review of the test of bio-equivalence-AUC, Cmax, and Tmax - will be given below in Section 2. The AUC, Cmax, and Tmax values for each GE drug are available from its package insert. Note that the package insert is referred to as the patient information leaflet in Europe (Schumann, 2006).

The use of GE drugs is often recommended to reduce medical costs, and pharmacists and/or medical doctors are asked to select a GE drug from among many candidates. However, it is not easy to do so since no means have been developed except for comparing their costs. In this paper, we develop a method that could be used for selecting better GE drugs from the list of candidates.

* Graduate School of Medicine, Kurume University

$\dagger$ Biostatistics Center, Kurume University

¥ Institute of Industrial Ecological Sciences, University of Occupational and Environmental Health, Japan

$\S$ Graduate School of Medicine, Kurume University 
We suppose in this paper that the characteristics of a drug are given by a subset of AUC, Cmax, and Tmax, and that the drug that has closer characteristics to the B-N drug is the better drug, and accordingly, the drug that has distant characteristics from the B-N drug is a poor drug. Note that the AUC, Cmax, and Tmax values for the GE and B-N drugs are fairly close within each trial, since GE drugs in the market have passed the test of bio-equivalence to the B-N drug, but often trial-to-trial variation in these values is substantial; for example, the AUC values for the $21 \mathrm{GE}$ drugs that will be discussed in the application section in this paper distribute in the range of 18.57 and 85.93 (see Table 7.1.). We develop a method to measure the closeness of the characteristics of the GE and B-N drugs, adjusting for trial-to-trial variation and also taking into account the property of the design used in bio-equivalence trials. A method is proposed to use the distance for selecting better GE drugs from the list of candidates.

In Section 2, we give a brief review of cross-over designs employed in trials of bioequivalence, and of the method of testing bio-equivalence. In Section 3, we introduce a mathematical model to represent $K$ trials of bio-equivalence taking into account trialto-trial variation. In Section 4, a method of estimating the distance of characteristics between the GE and B-N drugs is introduced. In Section 5, the ranking of GE drugs based on those distances and errors in ranking are discussed. A method of selecting better GE drugs is proposed in Section 6. Finally in Section 7 the proposed method is applied to 21 GE drugs that have passed bio-equivalence trials to Pravastatin, the B-N lipid-lowering compound.

\section{Trial of bio-equivalence}

The trial of bio-equivalence is conducted in a cross-over design. We briefly review the design and the trial of bio-equivalence in this section.

\subsection{Cross-over design}

The two-period two-treatment cross-over design is the standard design employed in a trial of bio-equivalence for drugs A and B. In this design, each subject receives drugs A and B. Half the subjects, called Group 1, receive A first and then, after a suitably chosen period of time that is called the wash-out period, receive B. The remaining subjects, called Group 2, receive B first and then A after the wash-out period. We briefly review the mathematical aspects of the design in this section. Readers unfamiliar with crossover designs are requested to refer to Fleiss (1986), or Kakuma and Hattori (2012).

Let $X_{i j}^{*}$ and $Y_{i j}^{*}$ be the data from the $j$-th subject who belongs to Group $i$ observed at the first treatment period and at the second treatment period, respectively $(i=1,2)$. The sizes of Groups 1 and 2 are assumed to be equal; it is denoted by $n$. These notations are summarized in Table 1.

Then, $X_{1 j}^{*}, X_{2 j}^{*}, Y_{1 j}^{*}$ and $Y_{2 j}^{*}$ may be represented as follows:

$$
\begin{aligned}
X_{1 j}^{*} & =\pi_{1}+\tau_{A}+\epsilon_{1 A j}, & & X_{2 j}^{*}=\pi_{1}+\tau_{B}+\epsilon_{1 B j}, \\
Y_{1 j}^{*} & =\pi_{2}+\tau_{B}+\epsilon_{2 B j}, & & Y_{2 j}^{*}=\pi_{2}+\tau_{A}+\epsilon_{2 A j},
\end{aligned}
$$

where $\pi_{\ell}$ is the effect parameter at treatment period $\ell(\ell=1,2) ; \tau_{A}$ and $\tau_{B}$ are the treatment effects of drugs $\mathrm{A}$ and $\mathrm{B}$, respectively; and $\left\{\epsilon_{1 A j}\right\}_{j=1}^{n},\left\{\epsilon_{1 B j}\right\}_{j=1}^{n},\left\{\epsilon_{2 A j}\right\}_{j=1}^{n}$, 
Table 1: AB/BA design

Representation of the data from the $j$-th subject in Groups 1 and 2

\begin{tabular}{c|cc|c}
\hline & \multicolumn{2}{|c|}{ Treatment Period } & \\
Treatment & 1 & 2 & Total \\
\hline Group 1 (A then B) & $X_{1 j}^{*}$ & $Y_{1 j}^{*}$ & $2 n$ \\
Group 2 (B then A) & $X_{2 j}^{*}$ & $Y_{2 j}^{*}$ & $2 n$ \\
\hline
\end{tabular}

and $\left\{\epsilon_{2 B j}\right\}_{j=1}^{n}$ are the error terms. These error terms are assumed to be mutually independent and identically distributed as a normal distribution with mean zero and variance $\sigma_{0}^{2}$.

Remark 1. $X_{i j}^{*}$ and $Y_{i j}^{*}$ often follow lognormal distributions. If this is the case $X_{i j}^{*}, \tau_{A}, Y_{i j}^{*}$ and $\tau_{B}$ in the above model are replaced, respectively by $\log X_{i j}^{*}, \log \tau_{A}$, $\log Y_{i j}^{*}$ and $\log \tau_{B}$.

Putting

$$
\begin{aligned}
\bar{X}_{i}^{*} & =\frac{1}{n} \sum_{j=1}^{n} X_{i j}^{*}, \quad \bar{Y}_{i}^{*}=\frac{1}{n} \sum_{j=1}^{n} Y_{i j}^{*}, \quad(i=1,2), \\
W_{A} & =\frac{1}{2}\left(\bar{X}_{1}^{*}+\bar{Y}_{2}^{*}\right), \quad W_{B}=\frac{1}{2}\left(\bar{X}_{2}^{*}+\bar{Y}_{1}^{*}\right), \\
S D_{A}^{2} & =\frac{1}{2(n-1)} \sum_{j=1}^{n}\left(\left(X_{1 j}^{*}-\bar{X}_{1}^{*}\right)^{2}+\left(Y_{2 j}^{*}-\bar{Y}_{2}^{*}\right)^{2}\right), \\
S D_{B}^{2} & =\frac{1}{2(n-1)} \sum_{j=1}^{n}\left(\left(X_{2 j}^{*}-\bar{X}_{2}^{*}\right)^{2}+\left(Y_{1 j}^{*}-\bar{Y}_{1}^{*}\right)^{2}\right),
\end{aligned}
$$

we have the following proposition, whose proof is straightforward and is omitted.

\section{Proposition 1}

(1) $E\left(W_{A}\right)=\tau_{A}+\left(\pi_{1}+\pi_{2}\right) / 2, E\left(W_{B}\right)=\tau_{B}+\left(\pi_{1}+\pi_{2}\right) / 2$,

(2) $V\left(W_{A}\right)=V\left(W_{B}\right)=\sigma_{0}^{2} /(2 n)$,

(3) $E\left(S D_{A}^{2}\right)=E\left(S D_{B}^{2}\right)=\sigma_{0}^{2}$.

Inspecting the proposition we put $X_{A}=W_{A} / \sigma_{0}, X_{B}=W_{B} / \sigma_{0}, N=2 n, \theta=$ $\left(\tau_{A}+2^{-1}\left(\pi_{1}+\pi_{2}\right)\right) / \sigma_{0}$, and $\delta=\left(\tau_{B}-\tau_{A}\right) / \sigma_{0}$, then $X_{A}$ and $X_{B}$ can be represented as follows:

$$
X_{A}=\theta+\epsilon_{A}, \quad X_{B}=\theta+\delta+\epsilon_{B},
$$

where $\epsilon_{A}$ and $\epsilon_{B}$ are independent and identically distributed random variables that follow a normal distribution with mean zero and variance $N^{-1}$. Parameter $\delta$, the standardized difference of treatment effects, is our main focus, but $\theta$ is a nuisance parameter whose interpretation is not easy.

Remark 2. Values of $\left(W_{A}, S D_{A}\right)$ and $\left(W_{B}, S D_{B}\right)$ are only available from the package insert. 


\subsection{Testing bio-equivalence}

Various procedures are employed in testing the bio-equivalence of drugs, depending on the type of drug (Center for Drug Evaluation and Research, 2003). We consider a typical procedure that may be illustrated as follows. Effects of GE and B-N drugs are compared in the two-period two-treatment cross-over design that uses healthy volunteers as subjects. Blood samples are taken at series of time points subsequent to dosing and assayed to determine drug concentration in the blood plasma. For each subject and period, drug concentrations in the blood plasma are plotted against the corresponding sampling times to give a concentration-time profile for that subject and period. The area under the profile is called the area under the curve (AUC), the peak of the curve is called the maximum concentration $(\mathrm{Cmax})$ and the time to the Cmax is called the maximum drug concentration time (Tmax). If we assume models given in (1) and (2) for AUC, then $\tau_{A}$ and $\tau_{B}$ tend to be the mean AUC of drugs A and B, respectively.

Now, drugs $\mathrm{A}$ and $\mathrm{B}$ are considered bio-equivalent if $0.8<\tau_{B} / \tau_{A}<1.25$ (Center for Drug Evaluation and Research, 2003), which is equivalent to $-\log 1.25<\log \tau_{B}-$ $\log \tau_{A}<\log 1.25$. AUC and Cmax often follow lognormal distributions, and the $90 \%$ confidence interval of $\log \tau_{B}-\log \tau_{A}$ is constructed based on $\log$ transformed data. Drugs A and B are judged to be bio-equivalent if the confidence interval is included in $(-\log 1.25, \log 1.25)$ (Center for Drug Evaluation and Research, 2003).

\section{Mathematical model}

Suppose that there are $K$ GE drugs developed for a B-N drug, which means that there are $K$ trials of bio-equivalence to the B-N drug. We consider $H(\leq 3)$ characteristics of a drug that consists of a subset of AUC, Cmax, and Tmax. As above, we assume for the $i$-th trial that the sizes of Group 1 and 2 are equal (which we denote by $n_{i}$, and put $\left.N_{i}=2 n_{i}\right)$, and that $\left(W_{A h i}, S D_{A h i}\right)$ and $\left(W_{B h i}, S D_{B h i}\right)$ are the values of the $h$-th characteristics of drugs A and B and their standard deviations obtained from the package inserts $(i=1, \ldots K)$. Putting $\sigma_{0 h i}=\left(S D_{A h i}+S D_{B h i}\right) / 2$, we assume similarly as the meta-analysis (van Houwelingen et al., 2002) that $\sigma_{0 h i}, i=1, \ldots K ; h=1, \ldots H$ are given constants. Since the scales of the drug characteristics are different, for example, AUC is measured in ng.hr/ml, while Tmax is measured in hours, we standardize these measurements by dividing each by its standard deviation: namely, we consider $X_{A h i}=W_{A h i} / \sigma_{0 h i}$ and $X_{B h i}=W_{B h i} / \sigma_{0 h i}$. Note that A corresponds to the B-N drug and $\mathrm{B}$ to the $i$-th GE drug.

Now, we introduce a model that takes into account trial-to-trial variation. Let $V_{h i}$ be a specific variable to the $i$-th trial and $\delta_{h i}$ be the unknown parameter representing the standardized difference of the $h$-th characteristic of the $i$-th GE and the B-N drugs, and let $\epsilon_{A h i}$ and $\epsilon_{B h i}$ be measurement errors. Then, model (3) in the previous section may be extended as follows:

$$
X_{A h i}=V_{h i}+\epsilon_{A h i}, \quad X_{B h i}=V_{h i}+\delta_{h i}+\epsilon_{B h i} .
$$

We assume that $\epsilon_{A h i}, h=1,2, \ldots, H, i=1,2, \ldots, K, \epsilon_{B h i}, h=1,2, \ldots, H$, and $i=1,2, \ldots, K$ are independent and identically distributed random variables following a distribution with mean zero and variance $1 / N_{i}$, and that vector $\mathbf{V}=\left(V_{1 i}, V_{2 i}, \ldots, V_{H i}\right)^{T}$ has mean $\Theta=\left(\theta_{1}, \theta_{2}, \cdots, \theta_{H}\right)^{T}$ and $H \times H$ covariance matrix $\Sigma_{0}$ whose $\left(h, h^{\prime}\right)$ element is denoted by $\sigma_{h h^{\prime}}$ if $h \neq h^{\prime}$ and by $\sigma_{h}^{2}$ if $h=h^{\prime}$. The model is similar to the random effect 
models used for meta-analysis in clinical trials that target the estimation of $\Theta$ (Berkey et al., 1995; Hardy and Thompson, 1996); however, our interest is in the estimation of $\delta_{h i}$.

\section{Estimation}

We do not assume specific distributions for $\mathbf{V}$ and $\epsilon^{\prime} s$, unable to apply the method of maximum likelihood, and develop an iterative method for estimating unknown parameters based on the method of weighted least squares in the next sections.

\subsection{Estimating $\Theta$ and $\left\{\delta_{h i}\right\}$ when $\Sigma_{0}$ is known}

We first assume that $\Sigma_{0}$ is known. Putting $Z_{0 i}=\left(X_{A 1 i}, X_{A 2 i}, \ldots, X_{A H i}\right)^{T}, Z_{1 i}=$ $\left(X_{B 1 i}, X_{B 2 i}, \ldots, X_{B H i}\right)^{T}$, and $Z_{i}=\left(Z_{0 i}^{T}, Z_{1 i}^{T}\right)^{T}$, it follows from (4) that $Z_{i}$ has the following mean vector and covariance matrix:

$$
\begin{aligned}
\Theta_{M i} & =\left(\begin{array}{c}
\Theta \\
\Theta
\end{array}\right)+\left(\begin{array}{c}
0 \\
\Delta_{i}
\end{array}\right), \\
\Sigma_{M i} & =\left(\begin{array}{cc}
\Sigma_{0} & \Sigma_{0} \\
\Sigma_{0} & \Sigma_{0}
\end{array}\right)+\frac{1}{N_{i}} I,
\end{aligned}
$$

where $\Theta$ is the mean of $V$ defined above, and $\Delta_{i}=\left(\delta_{1 i}, \delta_{2 i}, \ldots, \delta_{H i}\right)^{T}$ and $I$ is the $2 H$-dimensional unit matrix. We estimate $\Theta$ and $\Delta_{i}, i=1,2, \ldots, K$ by minimizing the sum of weighted squared deviations

$$
Q_{M}=\sum_{i=1}^{K}\left(Z_{i}-\Theta_{M i}\right)^{T} \Sigma_{M i}{ }^{-1}\left(Z_{i}-\Theta_{M i}\right)
$$

with respect to $\theta_{1}, \theta_{2}, \ldots, \theta_{H}$ and $\delta_{1 i}, \delta_{2 i}, \ldots, \delta_{H i}, i=1,2, \ldots, K$. The resulting weighted least squared estimates are given explicitly in the following theorem.

THEOREM 1. Assume that $\Sigma_{0}$ is a known non-singular $H \times H$ matrix, and put

$$
\left(\begin{array}{cc}
A_{1 i} & A_{2 i} \\
A_{2 i} & A_{3 i}
\end{array}\right)=\Sigma_{M i}^{-1}
$$

(1) Then, $A_{3 i}=A_{1 i}$ and the estimates of $\Theta$ and $\Delta_{i}, i=1,2, \ldots, K$ that minimize $Q_{M}$ are given by

$$
\begin{aligned}
\hat{\Theta} & =\left(\sum_{i=1}^{K}\left(A_{1 i}-A_{2 i} A_{1 i}^{-1} A_{2 i}\right)\right)^{-1} \sum_{i=1}^{K}\left(A_{1 i}-A_{2 i} A_{1 i}^{-1} A_{2 i}\right) Z_{0 i} \\
\hat{\Delta}_{i} & =A_{1 i}^{-1} A_{2 i}\left(Z_{0 i}-\hat{\Theta}\right)+\left(Z_{1 i}-\hat{\Theta}\right) .
\end{aligned}
$$

(2) $\hat{\Theta}$ and $\hat{\Delta}_{i}$ are unbiased estimates of $\Theta$ and $\Delta_{i}$

Proof. The proof of the theorem is given in Appendix.

\subsection{Estimating $\Theta$ and $\left\{\delta_{h i}\right\}$ when $\Sigma_{0}$ is unknown}

We first show two theorems. The proofs of the theorems are given in Appendix. 
Putting for $t=A, B$

$$
\begin{aligned}
S_{t h}^{2} & =\frac{1}{K-1} \sum_{i=1}^{K}\left(X_{t h i}-\bar{X}_{t h}\right)^{2}, \\
S_{A h, t h^{\prime}} & =\frac{1}{K-1} \sum_{i=1}^{K}\left(X_{A h i}-\bar{X}_{A h}\right)\left(X_{t h^{\prime} i}-\bar{X}_{t h^{\prime}}\right),
\end{aligned}
$$

we have the following theorem.

THEOREM 2.

(1) An unbiased estimator of $\sigma_{h}^{2}$ is given by

$$
\hat{\sigma}_{h}^{2}=\frac{1}{2}\left(S_{A h}^{2}-\frac{1}{K} \sum_{i=1}^{K} \frac{1}{N_{i}}+S_{A h, B h}\right) .
$$

(2) An unbiased estimator of $\sigma_{h h^{\prime}}\left(h \neq h^{\prime}\right)$ is given by

$$
\hat{\sigma}_{h h^{\prime}}=\frac{1}{3}\left(S_{A h, A h^{\prime}}+S_{A h, B h^{\prime}}+S_{A h^{\prime}, B h}\right) .
$$

THEOREM 3. Put

$$
\begin{aligned}
\bar{\delta}_{h} & =\frac{1}{K} \sum_{i=1}^{K} \delta_{h i}, \quad c_{\delta h}^{2}=\frac{1}{K-1} \sum_{i=1}^{K}\left(\delta_{h i}-\bar{\delta}_{h}\right)^{2}, \\
c_{\delta h, h^{\prime}} & =\frac{1}{K-1} \sum_{i=1}^{K}\left(\delta_{h i}-\bar{\delta}_{h}\right)\left(\delta_{h^{\prime} i}-\bar{\delta}_{h^{\prime}}\right) .
\end{aligned}
$$

Then, it follows that

$$
E\left(S_{B h}^{2}\right)=\sigma_{h}^{2}+K^{-1} \sum_{i=1}^{K} N_{i}^{-1}+c_{\delta h}^{2}, \quad E\left(S_{B h, B h^{\prime}}\right)=\sigma_{h h^{\prime}}+c_{\delta h, h^{\prime}} .
$$

When $\Sigma_{0}$ is unknown, we may plug in unbiased, and also consisitent, estimators given in Theorem 2 for $\Sigma_{0}$. In order to improve it, we propose in this section the following 2 -step estimator for estimating unknown parameters. The method is described as follows.

Step 1. Compute $\Sigma_{M i}$ in (5) by replacing $\sigma_{h}^{2}$ and $\sigma_{h h^{\prime}}$ in $\Sigma_{0}$ with their unbiased estimates $\hat{\sigma}_{h}^{2}$ and $\hat{\sigma}_{h h^{\prime}}$ that are given in Theorem 2 .

Step 2. Compute $\hat{\Theta}=\left(\hat{\theta}_{1}, \hat{\theta}_{2}, \ldots \hat{\theta}_{H}\right)$ and $\hat{\Delta}_{i}=\left(\hat{\delta}_{1 i}, \hat{\delta}_{2 i}, \ldots, \hat{\delta}_{H i}\right), i=1,2, \ldots, K$ using Theorem 1.

Step3. Compute $c_{\delta h}^{2}$ and $c_{\delta h, h^{\prime}}$ that are defined in Theorem 3 by replacing $\delta_{h i}$ with $\hat{\delta}_{h i}$, and then compute

$$
\tilde{\sigma}_{h}^{2}=\frac{1}{3}\left(S_{A h}^{2}+S_{B h}^{2}+S_{A h, B h}-\frac{2}{K} \sum_{i=1}^{K} \frac{1}{N_{i}}-c_{\delta h}^{2}\right),
$$




$$
\tilde{\sigma}_{h h^{\prime}}=\frac{1}{4}\left(S_{A h, A h^{\prime}}+S_{A h, B h^{\prime}}+S_{A h^{\prime}, B h}+S_{B h, B h^{\prime}}-c_{\delta h, h^{\prime}}\right),
$$

which are consistent estimates of $\sigma_{h}^{2}$ and $\sigma_{h h^{\prime}}$ from Theorems 2 and 3.

Step 4. Go to Step 1 using $\tilde{\sigma}_{h}^{2}$ and $\tilde{\sigma}_{h h^{\prime}}$, and then to Step 2 and 3. Repeat this process twice.

\subsection{Precision of the 2-step estimator}

We check the precision of the 2-step estimator by using the practical data give in Table 5 and by assuming normal distributions. If we assume normal distribution for $V^{\prime} s$ and $\epsilon^{\prime} s$, then we may estimate $\Theta$ and $\delta_{h i}$ by the method of maximum likelihood (mle). Table 2 gives the estimates of 1-step, 2-step estimators and mle obtained from the data given in Table 5, where the 1-step estimator is the estimator directory obtained from Step 1 and 2, without repetition. The fifth and sixth column of the table shows the absolute value of the difference of the two estimates from the mle, showing that the 2 -step estimator improves the precision of the 1-step estimator, actually, $\sum_{i=1}^{21} \mid 2$-step $m l e \mid=0.567$ and $\sum_{i=1}^{21} \mid 1$-step $-m l e \mid=0.710$; and that the 2 -step estimator is fairly close to the mle. Although this is only an example, it indicates that the 2-step estimators are comparable to mle even if the normality holds true.

A reviewer of the present paper raised a question of why not using the estimator that is converged in the iterative algorithm. We may, of course use it, but we find after a few simulation that the precision of the 2-step estimator is enough for our method of selecting better GE drugs.

\subsection{Comparison with the crude estimator for estimating $\delta_{h i}$}

Models given in (4) show that $V_{h i}$ is the factor for adjusting for the trial to trial variability. If we subtract $X_{A h i}$ from $X_{B h i}$ we may eliminate the factor and get an unbiased estimator for $\delta_{h i}$ that does not take into account the trial to trilal valiability. We call it the crude estimator of $\delta_{h i}$. The following theorem shows the superiority of $\hat{\delta}_{h i}$ to the crude estimator. Since we consider it for any given $h$, we drop $h$ from those suffixes for simplicity. Note that

$$
\operatorname{Var}\left(X_{B i}-X_{A i}\right)=\operatorname{Var}\left(\epsilon_{B i}-\epsilon_{A i}\right)=2 \frac{1}{N_{i}} .
$$

THEOREM 4. For $\sigma_{1}^{2}$, the variance of $X_{A i}$ (and also of $X_{B i}$ ), put

$$
\rho_{i}=\frac{\sigma_{1}^{2}}{\sigma_{1}^{2}+N_{i}^{-1}} .
$$

and assume that $\sigma_{1}^{2}$ is known. Then it follows that

$$
\operatorname{Var}\left(X_{B i}-X_{A i}\right)=\operatorname{Var}\left(\hat{\delta}_{i}\right)+\frac{\left(1-\rho_{i}\right)}{N_{i}}\left(1-\frac{\rho_{i}}{\sum_{j=1}^{k} \rho_{j}}\right)
$$

and that $\operatorname{Var}\left(\hat{\delta}_{i}\right)<\operatorname{Var}\left(X_{B i}-X_{A i}\right)$.

Proof. The proof of the theorem is given in Appendix. 
Table 2: Estimates of 1-step, 2-step estimators and mle from data in Table 5.

\begin{tabular}{c|ccccc}
\hline & \multicolumn{3}{|c}{ AUC } & \multicolumn{3}{c}{ absolute difference } \\
Drugs & 1-step & 2 -step & mle* & $\mid$ 1-step-mle $\mid$ & $\mid 2$-step-mle $\mid$ \\
\hline A & -0.014 & -0.014 & -0.013 & 0.001 & 0.001 \\
B & -0.119 & -0.137 & -0.184 & 0.064 & 0.046 \\
C & 0.156 & 0.154 & 0.158 & 0.002 & 0.004 \\
D & -0.038 & -0.032 & -0.008 & 0.030 & 0.025 \\
E & -0.226 & -0.236 & -0.266 & 0.041 & 0.030 \\
F & 0.071 & 0.071 & 0.063 & 0.008 & 0.008 \\
G & -0.117 & -0.144 & -0.238 & 0.121 & 0.094 \\
H & 0.136 & 0.138 & 0.137 & 0.001 & 0.001 \\
I & 0.060 & 0.063 & 0.070 & 0.010 & 0.007 \\
J & -0.064 & -0.065 & -0.073 & 0.009 & 0.008 \\
K & -0.180 & -0.174 & -0.149 & 0.031 & 0.026 \\
L & 0.127 & 0.135 & 0.177 & 0.050 & 0.041 \\
M & -0.021 & -0.022 & -0.031 & 0.010 & 0.009 \\
N & 0.011 & 0.024 & 0.073 & 0.062 & 0.048 \\
O & 0.055 & 0.056 & 0.059 & 0.004 & 0.003 \\
P & -0.180 & -0.180 & -0.164 & 0.017 & 0.016 \\
Q & 0.080 & 0.084 & 0.094 & 0.014 & 0.010 \\
R & 0.256 & 0.257 & 0.251 & 0.004 & 0.006 \\
S & 0.267 & 0.295 & 0.411 & 0.144 & 0.115 \\
T & -0.078 & -0.091 & -0.141 & 0.063 & 0.050 \\
U & -0.201 & -0.205 & -0.225 & 0.024 & 0.020 \\
\hline
\end{tabular}

*: mle is obtained under the assumption of normality.

\section{Ranking GE drugs}

\subsection{Distance of a GE drug from the B-N drug}

Define the distance between the $h$-th characteristic of the $i$-th GE and B-N drugs by $T_{h i}=\left|\hat{\delta}_{h i}\right|$ using the estimated $\hat{\delta}_{h i}$ in the preceding section. Arranging them in an ascending order, we may rank the GE drugs by means of the $h$-th characteristic, $h=1,2, \ldots, H$. One might expect a $\mathrm{GE}$ drug with a smaller rank to be better. However, this is not straightforward, since, as is shown in the next subsection, the nearest two ranks could be easily reversed. Alternatively, we may use $T_{i}=\left(\hat{\delta}_{1 i}^{2}+\hat{\delta}_{2 i}^{2}+\cdots+\hat{\delta}_{H i}^{2}\right)^{-1 / 2}$ for distance. However, we concentrate on the former distance in this paper to deal with the error in ranking.

\section{2. $\quad$ Error probability in ranking}

Suppose that there are two GE drugs, say F and G. Suppose also that we judge the rank of $\mathrm{F}$ to be smaller than the rank of $\mathrm{G}$ if $\hat{\delta}_{G}-\hat{\delta}_{F}>a$ for some constant $a>0$, where $\hat{\delta}_{F}\left(\hat{\delta}_{G}\right)$ is the estimate of $\delta_{F}\left(\delta_{G}\right)$. Then, an error probability of the judgment is expressed by

$$
\mathrm{EP}=P\left(\hat{\delta}_{G}-\hat{\delta}_{F}>a \mid \delta_{F} \geq \delta_{G}\right)
$$


We have the following theorem whose proof is given in Appendix.

THEOREM 5. If we assume normal distributions for $\epsilon^{\prime} s$ and $V^{\prime} s$ in model (4) and suppose $N=N_{F}=N_{G}$, then the error probability is represented by

$$
E P=1-\Phi\left(\frac{a+\left(\delta_{F}-\delta_{G}\right)}{\sqrt{2 \sigma_{1}^{2}(1-\rho)^{2}+2(1 / N)(1+\rho)^{2}}}\right)
$$

where $\rho=\rho_{F}\left(=\rho_{G}\right)$ is defined in Theorem 4, and $\Phi$ is the cumulative distribution function of a standard normal distribution.

Table 3 gives the values of the error probability for selected values of $a, \delta_{F}-\delta_{G}$ and $\sigma_{1}^{2}$. The table shows that the error probability in the ranking of two proximate GE drugs is substantial when values of $\delta_{F}-\delta_{G}$ and $a$ are small, but that it decreases as $\delta_{F}-\delta_{G}$ increases; for example, $E P=0.03$ when $\delta_{F}-\delta_{G}=1.0, a=0.2$, and $N_{1}=N_{2}=20$, indicating that the error probability of reversing the order of two drugs that have distant ranks is small.

Table 3: Probabilities of reversing ranks of two drugs when $N_{1}=N_{2}=20$

\begin{tabular}{c|cccc}
\hline & \multicolumn{2}{|c}{$\sigma_{1}^{2}=0.1$} & \multicolumn{2}{c}{$\sigma_{1}^{2}=5.0$} \\
$\delta_{F}-\delta_{G}$ & $a=0.1$ & $a=0.2$ & $a=0.1$ & $a=0.2$ \\
\hline 0.1 & 0.36 & 0.29 & 0.38 & 0.35 \\
0.3 & 0.23 & 0.18 & 0.26 & 0.24 \\
0.6 & 0.10 & 0.07 & 0.13 & 0.12 \\
1.0 & 0.02 & 0.01 & 0.04 & 0.03 \\
\hline \multicolumn{5}{c}{ Computed by equation (6). }
\end{tabular}

\subsection{A simulation for checking the stability of ranks}

A simulation is conducted to check the stability of ranks of the GE drugs when $K=10, H=1$ and $N_{i}=30,40, i=1,2, \ldots, K$. One thousand sets of $X_{A i}, X_{B i}$, $i=1,2, \ldots, K$ were generated from model (4). More precisely, random digits that follow a normal distribution with mean zero and variance $1 / N_{i}$ were generated and used for $\epsilon_{A i}$ 's and $\epsilon_{B i}$ 's, and random digits that follow normal and lognormal distributions with common mean $\theta$ and common standard deviation $\sigma=0.5,1,3$ were generated, and used for $V$ 's in the model. We call the former the normal model, and the latter the lognormal model. It follows from the condition of bio-equivalence that $\theta$ and $\delta_{i}$ must satisfy inequality $-0.25\left(\theta-\sigma_{0} \bar{\pi}\right)<\delta_{i}<0.25\left(\theta-\sigma_{0} \bar{\pi}\right)$, where $\bar{\pi}=\left(\pi_{1}+\pi_{2}\right), \sigma_{0}, \pi_{1}$, and $\pi_{2}$ are quantities defined in Section 2.1. Although $\bar{\pi}$ is unknown, we take this inequality into account and set $\theta=5$, sufficiently larger than $\delta_{i}$, where three sets of $\delta_{i}$ 's $\left(\delta_{i}=0.2 i\right.$, $\left.i=1,2, \ldots, K ; \delta_{i}=0.15 i, i=1,2, \ldots, K ; \delta_{i}=0.1 \ell, i=1,2, \ldots, K\right)$ were considered.

We use $r(F ; s)$ to denote the ranks of drug $\mathrm{F}$ in the $s$-th run, and consider the ranks of $\mathrm{F}$ in the $r$-th and $s$-th runs to agree iff $r(F ; s)=r(F ; t)$. We also define the rate of agreement as the sum of agreements of all GE drugs over a thousand runs divided by 1,000. Table 4 lists the rates of agreement under the normal and lognormal models when $N_{i}=30,40, i=1,2, \ldots, K$. The table shows that the rates of agreement are approximately larger than $90 \%$ if the distance between the GE and B-N drugs is larger 
than 0.20 when $N_{i} \geq 40, i=1,2, \ldots, 10$; that rates of the normal and lognormal models are almost equal; and that the rate increases slightly as $\sigma$ increases.

Table 4: Rates of agreement of the ranks under the normal and lognormal models

\begin{tabular}{|c|c|c|c|c|c|c|}
\hline \multirow[b]{3}{*}{$\delta$} & \multicolumn{6}{|c|}{$N_{i}=30, \quad i=1,2, \ldots, 10$} \\
\hline & \multicolumn{3}{|c|}{ Normal model } & \multicolumn{3}{|c|}{ Lognormal model } \\
\hline & $\sigma=0.5$ & $\sigma=1$ & $\sigma=3$ & $\sigma=0.5$ & $\sigma=1$ & $\sigma=3$ \\
\hline 0.20 & 0.87 & 0.98 & 1.00 & 0.86 & 0.97 & 1.00 \\
\hline 0.15 & 0.77 & 0.93 & 0.97 & 0.78 & 0.93 & 0.98 \\
\hline \multirow[t]{3}{*}{0.10} & 0.61 & 0.78 & 0.87 & 0.61 & 0.78 & 0.86 \\
\hline & \multicolumn{6}{|c|}{$N_{i}=40, \quad i=1,2, \ldots, 10$} \\
\hline & \multicolumn{3}{|c|}{ Normal model } & \multicolumn{3}{|c|}{ Lognormal model } \\
\hline$\delta$ & $\sigma=0.5$ & $\sigma=1$ & $\sigma=3$ & $\sigma=0.5$ & $\sigma=1$ & $\sigma=3$ \\
\hline$\overline{0.20}$ & 0.93 & 0.99 & 1.00 & 0.94 & 0.99 & 1.00 \\
\hline 0.15 & 0.88 & 0.98 & 1.00 & 0.87 & 0.98 & 1.00 \\
\hline 0.10 & 0.73 & 0.89 & 0.95 & 0.74 & 0.90 & 1.00 \\
\hline
\end{tabular}

\section{Method for selecting better GE drugs}

Findings in the preceeding section indicate that the direct use of ranks of GE drugs for selecting better one could run a risk, but the reverse probability of two GE drugs are small when their ranks are stay aloof. In other words, the ranking is fairly stable if the distance of two drugs is larger than 0.20 . We introduce in this section a method for selecting better GE drugs by taking into account these findings. The method is as follows.

- Classify GE drugs into three groups as follows by using estimated AUC distances from the B-N drug. (1) Take $a_{0}=\left(\right.$ the second largest $\left\{\hat{\delta}_{i}, i=1, \ldots, K\right\}$ - smallest $\left.\left\{\hat{\delta}_{i}, i=1, \ldots, K\right\}\right) / 3$. (2) Classify drugs into three groups as follows: the first group consists of drugs with AUC distances less than or equal to $a_{0}$, the second group consists of drugs with AUC distances larger than $a_{0}$ but less than or equal to $2 a_{0}$, and the third group consists of drugs with AUC distances larger than $2 a_{0}$. Denote these groups by $g_{1 a u c}, g_{2 a u c}$, and $g_{3 a u c}$, respectively.

- In addition, classify GE drugs by using Cmax distances in the same way and denote these groups as $g_{1 \text { cmax }}, g_{2 \text { cmax }}$, and $g_{3 c \max }$.

- Set $G_{1}=g_{1 a u c} \cap g_{1 c \max }, G_{3}=g_{3 a u c} \cap g_{3 c \max }$, and $G 2=$ \{other drugs , and select drugs in group $G_{1}$ as better GE drugs in the list of candidates.

The behavior of the method will be assessed in the next section by simulation. Note that the largest distance can be unusually large, and although we excluded it when defining the cut-off point, $a_{0}$, we include it in $G_{3}$ 


\section{Application}

\section{1. data}

Pravastatin is a lipid-lowering compound - a HMG-CoA reductase inhibitor, which reduces cholesterol biosynthesis. It is used for preventing cardiovascular diseases. We consider Pravastatin as the B-N drug. More than twenty generic drugs that are bioequivalent to Pravastatin are sold in the market. We consider twenty-one of them by naming them randomly as $A, B, \ldots, T, U$. Table 7.1. lists the values of AUC, Cmax, and Tmax, their standard deviations, and the size of the two groups in each trial that we collected from the package insert of each generic drug. We use AUC and Cmax as the characteristics of the drugs and apply our method; thus, $H=2$ and $K=21$.

Note that the variability of AUC among trials is tremendous, spreading from 18.42 $\mathrm{nghr} / \mathrm{ml}$ to $85.93 \mathrm{nghr} / \mathrm{ml}$. This is because of the Guideline (Center for Drug Evalu and Research 2003) onley states the key points of the sampling schedule of taking blood samples. The number of time intervals and times for the sampling are left to generic manifacturers. To check the validity of using such AUC in our model, we obtained the time of the last sampling point from each trial from the package insert and studied its correlation with the AUC. We find a significant relationship between the time of the last sampling point and $\left\{W_{A 1 i}\right\}$, but it disappeared when $X_{A 1 i}=W_{A 1 i} / S D_{A 1 i}$ is used instead of $W_{A 1 i}$. Thus the standardization is not only adjust for the scale of the drug characteristics, but also provides us the validity of applying our model given in (4).

\subsection{Application of the proposed method}

$\Sigma_{0}$ in Step 1 is computed by setting $H=2$, giving $\sigma_{1}^{2}=0.21, \sigma_{2}^{2}=0.17, \sigma_{12}=0.16$. Next, using these values for $\Sigma_{0}$ estimates of $\theta_{1}, \theta_{2}, \delta_{1 i}$, and $\delta_{2 i}$ are obtained by the method illustrated in Step 2. Then, $\Sigma_{0}$ is refined by using the method given in Step 3 . Finally, Step 4 is conducted by using the refined $\Sigma_{0}$. The results are summarized in Table 6. The first column of the table lists the id of the GE drugs, from the second to the fourth columns list the AUC results, and from the fifth to the seventh columns list the Cmax results. Note that the fourth and seventh columns list the grouping results of drugs by AUC and Cmax; and the last column list the results of the grouping of these drugs into three groups. The colums show that the agreement of groups made by AUC and Cmax is not good, for example, Drug E is classified into the group 3 by AUC, whereas it is classified into the group 1 by Cmax. The cut-off points of the first and second groups and of the second and third groups for AUC are 0.081 and 0.162, respectively, and for Cmax are 0.069 and 0.138 , respectively. The estimates of the other parameters are $\hat{\sigma}_{1}^{2}=0.21, \hat{\sigma}_{2}^{2}=0.17, \hat{\sigma}_{12}=0.16, \hat{\theta}_{1}=2.19$, and $\hat{\theta}_{2}=1.91$. It follows from Table 6 that $G_{1}=\{A, J, M, O\}, G_{2}=\{B, C, D, E, F, G, H, I, K, L, N, Q, S, T, U\}$, and $G_{3}=\{P, R\}$. From this result we recommend GE drugs in group $G 1$ as better GE drugs among the candidates.

\subsection{Checking the reliability}

We conducted a simulation to check the reliability of the obtained results. The framewok of the simulation is as follows. Assuming the estimated parameters as true parameters we generated one thousand sets of $X_{A h i}, X_{B h i}, i=1,2, \ldots, K(K=21)$ and $h=1,2$ from model (4) under the normal and lognormal models, applied the proposed 
Table 5: Values of AUC, Cmax, and Tmax, their standard deviations, and the size of the two groups in the 21 trials of bio-equivalence to Pravastatin

\begin{tabular}{|c|c|c|c|c|}
\hline & AUC: nghr/ml (SD) & Cmax: ng/ml (SD) & Tmax: hr (SD) & $\mathrm{n}$ \\
\hline $\mathrm{A}$ & $46.97(23.20)$ & $19.88(11.27)$ & $1.10(0.30)$ & 20 \\
\hline Pravastatin & $47.24(21.82)$ & $19.98(10.80)$ & $1.10(0.30)$ & 20 \\
\hline B & $27.43(12.49)$ & $12.41(4.56)$ & $1.10(0.20)$ & 14 \\
\hline Pravastatin & $28.07(8.53)$ & $13.11(4.43)$ & $1.10(0.20)$ & 14 \\
\hline $\mathrm{C}$ & $43.22(14.94)$ & $16.30(6.09)$ & $1.00(0.18)$ & 23 \\
\hline Pravastatin & $40.93(16.92)$ & $15.95(7.64)$ & $1.10(0.27)$ & 23 \\
\hline $\mathrm{D}$ & $36.39(17.07)$ & $14.01(8.02)$ & $1.20(0.30)$ & 19 \\
\hline Pravastatin & $37.56(14.63)$ & $14.31(6.91)$ & $1.20(0.50)$ & 19 \\
\hline $\mathrm{E}$ & $28.12(13.18)$ & $14.31(6.31)$ & $1.03(0.26)$ & 20 \\
\hline Pravastatin & $30.61(13.19)$ & $15.21(6.68)$ & $0.98(0.30)$ & 20 \\
\hline $\mathrm{F}$ & $44.10(25.10)$ & $18.70(12.40)$ & $1.20(0.40)$ & 28 \\
\hline Pravastatin & $41.90(24.60)$ & $17.00(11.50)$ & $1.20(0.30)$ & 28 \\
\hline $\mathrm{G}$ & $18.57(9.87)$ & $6.62(2.69)$ & $1.53(0.32)$ & 18 \\
\hline Pravastatin & $18.42(8.14)$ & $6.62(2.44)$ & $1.53(0.36)$ & 18 \\
\hline $\mathrm{H}$ & $38.49(25.07)$ & $17.99(14.42)$ & $1.04(0.31)$ & 60 \\
\hline Pravastatin & $35.25(20.49)$ & $16.68(12.84)$ & $1.13(0.40)$ & 60 \\
\hline I & 38.95 (19.99) & $17.49(10.99)$ & $1.10(0.40)$ & 20 \\
\hline Pravastatin & $37.74(20.16)$ & $17.74(12.54)$ & $1.10(0.20)$ & 20 \\
\hline $\mathrm{J}$ & $37.94(21.48)$ & $17.31(10.75)$ & $1.03(0.22)$ & 30 \\
\hline Pravastatin & $38.97(18.27)$ & $17.01(8.88)$ & $1.15(0.27)$ & 30 \\
\hline $\mathrm{K}$ & $73.22(30.63)$ & $21.02(11.62)$ & $1.10(0.20)$ & 30 \\
\hline Pravastatin & $80.35(38.18)$ & $21.40(11.39)$ & $1.20(0.30)$ & 30 \\
\hline $\mathrm{L}$ & $85.93(33.86)$ & $37.98(18.72)$ & $1.17(0.36)$ & 23 \\
\hline Pravastatin & $83.66(30.31)$ & $34.04(14.59)$ & $1.24(0.30)$ & 23 \\
\hline $\mathrm{M}$ & $53.20(29.15)$ & $18.32(11.80)$ & $1.20(0.30)$ & 20 \\
\hline Pravastatin & $53.20(28.86)$ & $18.81(11.85)$ & $1.20(0.30)$ & 20 \\
\hline $\mathrm{N}$ & $47.40(25.70)$ & $19.80(14.90)$ & $1.07(0.40)$ & 20 \\
\hline Pravastatin & $48.50(19.30)$ & $19.90(12.30)$ & $1.08(0.23)$ & 20 \\
\hline $\mathrm{O}$ & $33.88(17.24)$ & $17.87(11.35)$ & $1.10(0.30)$ & 24 \\
\hline Pravastatin & $33.00(14.63)$ & $17.86(8.89)$ & $1.10(0.30)$ & 24 \\
\hline $\mathrm{P}$ & $55.73(23.07)$ & $19.81(9.11)$ & $1.20(0.30)$ & 19 \\
\hline Pravastatin & $60.52(21.79)$ & $21.59(9.08)$ & $1.10(0.20)$ & 19 \\
\hline $\mathrm{Q}$ & $47.70(22.40)$ & $20.16(11.34)$ & $1.20(0.20)$ & 26 \\
\hline Pravastatin & $46.00(23.68)$ & $19.27(12.54)$ & $1.20(0.30)$ & 26 \\
\hline $\mathrm{R}$ & $51.60(32.85)$ & $19.90(14.00)$ & $1.10(0.54)$ & 29 \\
\hline Pravastatin & $44.30(21.00)$ & $15.70(9.15)$ & $1.30(0.54)$ & 29 \\
\hline $\mathrm{S}$ & $35.10(9.80)$ & $11.70(4.40)$ & $1.20(0.20)$ & 10 \\
\hline Pravastatin & $35.60(8.90)$ & $12.00(4.40)$ & $1.30(0.40)$ & 10 \\
\hline $\mathrm{T}$ & $49.80(26.70)$ & $23.10(11.60)$ & $1.40(0.40)$ & 20 \\
\hline Pravastatin & $49.90(27.80)$ & $22.50(11.50)$ & $1.40(0.40)$ & 20 \\
\hline $\mathrm{U}$ & $47.98(24.61)$ & $18.24(9.84)$ & $1.20(0.30)$ & 20 \\
\hline Pravastatin & $52.58(30.28)$ & $19.07(11.57)$ & $1.30(0.40)$ & 20 \\
\hline
\end{tabular}


Table 6: Estimated $\left|\delta_{i}\right|$ in AUC and Cmax of Pravastatin

\begin{tabular}{c|cccccc|c}
\hline & \multicolumn{3}{|c|}{ AUC } & \multicolumn{5}{c}{ Cmax } & \\
Drugs & $\left|\hat{\delta}_{i}\right|$ & rank & $g_{\text {auc }}$ & $\left|\hat{\delta}_{i}\right|$ & rank & $g_{\text {cmax }}$ & Group \\
\hline $\mathrm{A}$ & 0.014 & 1 & 1 & 0.020 & 2 & 1 & 1 \\
$\mathrm{M}$ & 0.022 & 2 & 1 & 0.065 & 7 & 1 & 1 \\
$\mathrm{~N}$ & 0.026 & 3 & 1 & 0.124 & 15 & 2 & 2 \\
$\mathrm{D}$ & 0.032 & 4 & 1 & 0.073 & 10 & 2 & 2 \\
$\mathrm{O}$ & 0.056 & 5 & 1 & 0.015 & 1 & 1 & 1 \\
$\mathrm{I}$ & 0.064 & 6 & 1 & 0.075 & 11 & 2 & 2 \\
$\mathrm{~J}$ & 0.065 & 7 & 1 & 0.026 & 3 & 1 & 1 \\
$\mathrm{~F}$ & 0.071 & 8 & 1 & 0.111 & 14 & 2 & 2 \\
$\mathrm{Q}$ & 0.084 & 9 & 2 & 0.036 & 4 & 1 & 2 \\
$\mathrm{~T}$ & 0.092 & 10 & 2 & 0.127 & 16 & 2 & 2 \\
$\mathrm{~L}$ & 0.136 & 11 & 2 & 0.198 & 19 & 3 & 2 \\
$\mathrm{H}$ & 0.138 & 12 & 2 & 0.068 & 9 & 1 & 2 \\
$\mathrm{~B}$ & 0.139 & 13 & 2 & 0.078 & 12 & 2 & 2 \\
$\mathrm{G}$ & 0.147 & 14 & 2 & 0.222 & 20 & 3 & 2 \\
$\mathrm{C}$ & 0.154 & 15 & 2 & 0.089 & 13 & 2 & 2 \\
$\mathrm{~K}$ & 0.174 & 16 & 3 & 0.065 & 8 & 1 & 2 \\
$\mathrm{P}$ & 0.179 & 17 & 3 & 0.161 & 18 & 3 & 3 \\
$\mathrm{U}$ & 0.206 & 18 & 3 & 0.063 & 6 & 1 & 2 \\
$\mathrm{E}$ & 0.237 & 19 & 3 & 0.045 & 5 & 1 & 2 \\
$\mathrm{R}$ & 0.257 & 20 & 3 & 0.324 & 21 & 3 & 3 \\
$\mathrm{~S}$ & 0.298 & 21 & 3 & 0.132 & 17 & 2 & 2 \\
\hline
\end{tabular}

method to each set and classified the GE drugs into the three groups. Table 7 summarizes the results of the simulation. The first column of the table lists the true groups; namely the obtained results given above. The second, third, and fourth columns list the average proportions of drugs selected for groups $G_{1}, G_{2}$, and $G_{3}$ by the proposed method under the normal model; and the fifth, sixth, and seventh columns list the corresponding proportions under the lognormal model. The table shows that proportions of the normal and lognormal models are almost equal; and that the proportion of the drugs in $G 1$ to be classified as $G 3$ drugs is 0.03 , showing a stability of the obtained results.

Table 7: Proportions of drugs in the true group selected as $G_{1}, G_{2}$, and $G_{3}$

\begin{tabular}{c|ccc|cccc}
\hline & \multicolumn{6}{|c}{ Selected } \\
\cline { 2 - 7 } True group & \multicolumn{3}{|c}{ Normal model } & \multicolumn{4}{c}{ Lognormal model } \\
\cline { 2 - 7 } & $G 1$ & $G 2$ & $G 3$ & $G 1$ & $G 2$ & $G 3$ \\
\hline$G 1$ & 0.25 & 0.71 & 0.04 & 0.26 & 0.71 & 0.03 \\
$G 2$ & 0.20 & 0.72 & 0.08 & 0.19 & 0.73 & 0.08 \\
$G 3$ & 0.09 & 0.72 & 0.19 & 0.10 & 0.70 & 0.20 \\
\hline
\end{tabular}




\section{Discussion}

It is widely misconceived that GE drugs are perfect copies of the B-N drug. However, from a statistical point of view, these drugs have only passed statistical tests of bio-equivalence conducted by using measurements of AUC and Cmax in the blood of healthy volunteers. Typically, GE drugs may differ in excipients with which the active principle is associated in the final drug product. The difference may influence dissolution rates in the gastrointestinal tract, and thus, absorption of the drug substance; more rapid absorption rates may be associated with a higher incidence of side effects (Olling et. al., 1999; Kramer et.al., 2007).

Apart from the isue on the excipients, the present study makes it clear that there are about 30 time difference between the smallest and the largest estimated distances regarding the AUC of Probastatin. Since those GE drugs have passed the test of bioequivalence, it is not easy to differentiate two drugs that have proximate ranks; actually, their confidence intervals heavily overlap. However, we find that there could be a substantial difference between the smallest and largest distances. The method proposend in this paper aimes to take advantage of this finding; it prevents the risk of G1 drugs to be judged as G3 drugs.

\section{Appendix}

\section{Proof of Theorem 1}

Let $A$ be a $p \times p$ matrix, and $b, x$, and $c$ be p-dimensional column vectors; then, we first note that it follows that

$$
\begin{aligned}
\frac{\partial}{\partial x}(b-x)^{T} A(b-x) & =-2 A(b-x), \\
\frac{\partial}{\partial x}(b-x)^{T} A(c-x) & =-A(b-x)-A(c-x), \\
\frac{\partial}{\partial x}(b-x)^{T} c & =\frac{\partial}{\partial x} c^{T}(b-x)=-c .
\end{aligned}
$$

Now applying these operations, we have

$$
\begin{aligned}
& \frac{\partial Q_{M}}{\partial \Theta}=0 \quad \Longleftrightarrow \\
& \sum_{i=1}^{K}\left(A_{1 i}\left(Z_{0 i}-\Theta\right)+A_{2 i}\left(Z_{0 i}-\Theta\right)+A_{2 i}\left(Z_{1 i}-\Theta\right)\right. \\
&\left.+A_{1 i}\left(Z_{1 i}-\Theta\right)-\left(A_{2 i}+A_{1 i}\right) \Delta_{i}\right)=0
\end{aligned}
$$

$$
\frac{\partial Q_{M}}{\partial \Delta_{i}}=0 \Longleftrightarrow A_{1 i} \Delta_{i}-A_{2 i}\left(Z_{0 i}-\Theta\right)-A_{1 i}\left(Z_{1 i}-\Theta\right)=0 .
$$

Since $\Sigma_{0}$ is assumed non-singular $A_{1 i}$ is non-singular and it follows from (8)

$$
\Delta_{i}=A_{1 i}^{-1} A_{2 i}\left(Z_{0 i}-\Theta\right)+\left(Z_{1 i}-\Theta\right) .
$$

Substituting (9) into (7), we have

$$
\sum_{i=1}^{K}\left(A_{1 i}-A_{2 i} A_{1 i}^{-1} A_{2 i}\right) Z_{0 i}=\sum_{i=1}^{K}\left(A_{1 i}-A_{2 i} A_{1 i}^{-1} A_{2 i}\right) \Theta,
$$


if $A_{1 i}$ is non-singular. Thus,

$$
\begin{aligned}
\hat{\Theta} & =\left(\sum_{i=1}^{K}\left(A_{1 i}-A_{2 i} A_{1 i}^{-1} A_{2 i}\right)^{-1} \sum_{i=1}^{K}\left(A_{1 i}-A_{2 i} A_{1 i}^{-1} A_{2 i}\right) Z_{0 i},\right. \\
\hat{\Delta}_{i} & =A_{3}^{-1} A_{2}\left(Z_{0 i}-\hat{\Theta}\right)+\left(Z_{1 i}-\hat{\Theta}\right) .
\end{aligned}
$$

\section{Proof of Theorem 2 and 3}

Since

$$
\begin{aligned}
S_{A h}^{2} & =\frac{1}{K-1}\left(\sum_{i=1}^{K} X_{A h i}^{2}-K \bar{X}_{A h}^{2}\right), \\
E\left(\sum_{i=1}^{K} X_{A h i}^{2}\right) & =\sum_{i=1}^{K}\left(\operatorname{Var}\left(X_{A h i}\right)+\left(E\left(X_{A h i}\right)\right)^{2}\right)=K\left(\sigma_{h}^{2}+\theta_{h}^{2}\right)+\sum_{i=1}^{K} N_{i}^{-1}, \\
E\left(\bar{X}_{A h}^{2}\right) & =\operatorname{Var}\left(\bar{X}_{A h}\right)+\left(E\left(\bar{X}_{A h}\right)\right)^{2}=\frac{1}{K^{2}} \sum_{i=1}^{K}\left(\sigma_{h}^{2}+N_{i}^{-1}\right)+\theta_{h}^{2},
\end{aligned}
$$

we have

$$
E\left(S_{A h}^{2}\right)=\sigma_{h}^{2}+\frac{1}{K} \sum_{i=1}^{K} N_{i}^{-1} .
$$

Similarly as above, we can show that

$$
E\left(S_{A h, B h}\right)=\sigma_{h}^{2}, \quad E\left(S_{A h, A h^{\prime}}\right)=E\left(S_{A h, B h^{\prime}}\right)=\sigma_{h h^{\prime}} .
$$

We get Theorem 2 from these equations.

Now, similarly as above we may show

$$
\begin{aligned}
E\left(S_{B h}^{2}\right) & =\sigma_{h}^{2}+\frac{1}{K} \sum_{i=1}^{K} N_{i}^{-1}+\frac{1}{K-1} \sum_{i=1}^{K}\left(\delta_{h i}-\bar{\delta}_{h}\right)^{2}, \\
E\left(S_{B h, B h^{\prime}}\right) & =\sigma_{h h^{\prime}}+\frac{1}{K-1} \sum_{i=1}^{K}\left(\delta_{h i}-\bar{\delta}_{h}\right)\left(\delta_{h^{\prime} i}-\bar{\delta}_{h^{\prime}}\right) .
\end{aligned}
$$

Thus the proof of Theorem 3 is immediate by these equations.

\section{Proof of Theorem 4}

Since $Q_{M}$ may be represented as

$$
\begin{array}{r}
Q_{M}=\frac{1}{\sigma_{1}^{2}} \sum_{k=1}^{K} \frac{\rho_{k}}{1-\rho_{k}^{2}}\left(\left(X_{A k}-\theta\right)^{2}-2 \rho_{k}\left(X_{A k}-\theta\right)\left(X_{B k}-\theta-\delta_{k}\right)\right. \\
\left.+\left(X_{B k}-\theta-\delta_{k}\right)^{2}\right),
\end{array}
$$

we have

$$
\hat{\theta}=\frac{\sum_{k=1}^{K} \rho_{k} X_{A k}}{\sum_{k=1}^{K} \rho_{k}},
$$




$$
\hat{\delta}_{k}=X_{B k}-\hat{\theta}-\rho_{k}\left(X_{A k}-\hat{\theta}\right)
$$

where

$$
\rho_{k}=\frac{\sigma_{1}^{2}}{\sigma_{1}^{2}+N_{k}^{-1}} .
$$

The variance of $\hat{\delta}_{k}$ is represented as

$$
\begin{array}{r}
V\left(\hat{\delta}_{k}\right)=V\left(X_{B k}\right)+\rho_{k}^{2} V\left(X_{A k}\right)+\left(1-\rho_{k}\right)^{2} V(\hat{\theta})-2 \rho_{k} \operatorname{cov}\left(X_{B k}, X_{A k}\right) \\
-2\left(1-\rho_{k}\right) \operatorname{cov}\left(X_{B k}, \hat{\theta}\right)+2 \rho_{k}\left(1-\rho_{k}\right) \operatorname{cov}\left(X_{A k}, \hat{\theta}\right) .
\end{array}
$$

Now we may show

$$
\begin{aligned}
& V\left(X_{B k}\right)=V\left(X_{A k}\right)=\sigma_{1}^{2}+N_{k}^{-1}, \operatorname{cov}\left(X_{B k}, X_{A k}\right)=\sigma_{1}^{2}, \\
& \operatorname{cov}\left(X_{B k}, \hat{\theta}\right)=\frac{\rho_{k}}{\sum_{k=1}^{K} \rho_{k}} \sigma_{1}^{2} \text { and } \operatorname{cov}\left(X_{A k}, \hat{\theta}\right)=\frac{1}{\sum_{k=1}^{K} \rho_{k}} \sigma_{1}^{2},
\end{aligned}
$$

and since $\sigma_{1}^{2}+k_{k}^{-1}=\sigma_{1}^{2} / \rho_{k}$ from (11), we have

$$
V(\hat{\theta})=\frac{1}{\left(\sum_{k=1}^{K} \rho_{k}\right)^{2}} \sum_{k=1}^{K} \rho_{k}^{2}\left(\sigma_{1}^{2}+N_{k}^{-1}\right)=\frac{\sigma_{1}^{2}}{\sum_{k=1}^{K} \rho_{k}} .
$$

Thus substituting these equations to (12), it follows that

$$
V\left(\hat{\delta}_{k}\right)=\left(1+\rho_{k}^{2}\right)\left(\sigma_{1}^{2}+N_{k}^{-1}\right)+\left(1-\rho_{k}\right)^{2} \frac{\sigma_{1}^{2}}{\sum_{k=1}^{K} \rho_{k}}-2 \rho_{k} \sigma_{1}^{2} .
$$

Thus

$$
V\left(X_{B k}-X_{A k}\right)-V\left(\hat{\delta}_{k}\right)=\frac{1-\rho_{k}^{2}}{N_{k}}-\sigma_{1}^{2}\left(1-\rho_{k}\right)^{2}\left(1+\frac{1}{\rho_{i}}\right) .
$$

Again we have $\sigma_{1}^{2}\left(1-\rho_{k}\right)=\rho_{k} / N_{k}$ from (11), thus substituting it to the above equation we finally have

$$
V\left(X_{B k}-X_{A k}\right)-V\left(\hat{\delta}_{k}\right)=\left(1-\rho_{k}\right) \frac{1}{N_{k}}\left(1-\frac{\rho_{k}}{\sum_{i=1}^{K} \rho_{i}}\right) .
$$

This completes the proof of Theorem 4 .

\section{Proof of Theorem 5}

It follows from (10) that

$$
\hat{\delta}_{G}-\hat{\delta}_{F}=\left(X_{B G}-\rho_{G} X_{A G}\right)-\left(X_{B F}-\rho_{F} X_{A F}\right)+\left(\rho_{G}-\rho_{F}\right) \hat{\theta} .
$$

Thus

$$
\begin{aligned}
& V\left(\hat{\delta}_{G}-\hat{\delta}_{F}\right)=V\left(X_{B G}-\rho_{G} X_{A G}\right)+V\left(X_{B F}-\rho_{F} X_{A F}\right)+\left(\rho_{G}-\rho_{F}\right)^{2} V(\hat{\theta}) \\
& -2 \operatorname{cov}\left(X_{B G}-\rho_{G} X_{A G}, X_{B F}-\rho_{F} X_{A F}\right)+2\left(\rho_{G}-\rho_{F}\right) \operatorname{cov}\left(X_{B G}-\rho_{G} X_{A G}, \hat{\theta}\right) \\
& -2\left(\rho_{G}-\rho_{F}\right) \operatorname{cov}\left(X_{B F}-\rho_{F} X_{A F}, \hat{\theta}\right)
\end{aligned}
$$




$$
=V\left(X_{B G}-\rho_{G} X_{A G}\right)+V\left(X_{B F}-\rho_{F} X_{A F}\right)+\left(\rho_{G}-\rho_{F}\right)^{2} V(\hat{\theta}),
$$

since

$$
\begin{array}{r}
\operatorname{cov}\left(X_{B G}-\rho_{G} X_{A G}, X_{B F}-\rho_{F} X_{A F}\right)=\operatorname{cov}\left(X_{B G}-\rho_{G} X_{A G}, \hat{\theta}\right) \\
=\operatorname{cov}\left(X_{B F}-\rho_{F} X_{A F}, \hat{\theta}\right)=0
\end{array}
$$

Furthermore, we may show

$$
V\left(X_{B i}-\rho_{i} X_{A i}\right)=\sigma_{1}^{2}\left(1-\rho_{i}\right)^{2}+N_{i}^{-1}\left(1+\rho_{i}^{2}\right)
$$

for $i=G, F$, and it folows that

$$
\begin{array}{r}
V\left(\hat{\delta}_{G}-\hat{\delta}_{F}\right)=\sigma_{1}^{2}\left(1-\rho_{G}\right)^{2}+N_{G}^{-1}\left(1+\rho_{G}^{2}\right)+\sigma_{1}^{2}\left(1-\rho_{F}\right)^{2} \\
+N_{F}^{-1}\left(1+\rho_{F}^{2}\right)+\left(\rho_{G}-\rho_{F}\right)^{2} \frac{\sigma_{1}^{2}}{\sum_{k=1}^{K} \rho_{k}} .
\end{array}
$$

In particular, when $N_{G}=N_{F}=N$ we have $\rho_{G}=\rho_{F}=\rho$ and

$$
V\left(\hat{\delta}_{G}-\hat{\delta}_{F}\right)=2 \sigma_{1}^{2}(1-\rho)^{2}+2 N^{-1}(1+\rho)^{2} .
$$

Since the normality is assumed for $V$ 's and $\epsilon_{A}$ 's and $\epsilon_{B}$ 's, rest of the proof of the theorem is straightforward.

\section{References}

Berkey, C. S., Hoaglin, D. C., Mosteller, F. and Coldditz, G. A. (1995). A randomeffects regression model for meta-analysis. Statistics in Medicine 14, 395-411.

Center for Drug Evaluation and Research (2003). Guidance for Industry: Bioavailability and Bioequivalence Studies for Orally Administered Drug Products, General Considerations. United States Food and Drug Administration. http://www.fda.gov/downloads/Drugs/GuidanceComplianceRegulatoryInformation /Guidances/ucm070.

Fleiss, J. L. (1996). The Design and Analysis of Clinical Experiments, J. Wiley and Sons, New York.

Hardy, R. J., Thompson, S. G. (1996). A likelihood approach to meta-analysis with random effects. Statistics in Medicine 15, 619-629.

Kakuma, T. and Hattori, S. (2012). Rinshousiken no Dezain to Kaiseki (in Japanese), Baio-toukei seri-zu, Kindaikagaku-sha, Tokyo.

Kramer, G., Biraben, A., Carreno, M., Guekht, A., de Haan, G. J., Jedrezejczak, J., Josephs, D. van Rijckevorsel, K. and Zaccara, G. (2007). Current approaches to the use of generic antiepiletic drugs. Epilepsy \& Behavior 11, 46-52.

Olling, M., Mensinga, T. T., Barends, D. M., Groen, C., Lake, O. A. and Meulenbelt, J. (1999). Bioavailability of carbamazepine from four different products and the occurrence of side effects. Biopharmaceutics Drug Disposition 20, 19-28.

Schumann, B. M. (2006). FDA Regulation of Patient Package Insert. Chadbourne \& 
Parkllp. Available at: http://bit.ly/138Uq45 (accessed December 20, 2011)

van Houwelingen, H. C., Arends, L. R. and Stijinen, T. (2002). Advanced methods in meta-analysis: multivariate approach and meta-regression. Statistics in Medicine 21 (4), 589-624.

Received August 6, 2014

Revised December 4, 2014 